\title{
A Reliable Channel Reservation based Multi-channel MAC Protocol with A Single Transceiver
}

\author{
(Invited Paper) \\ Bo Yang, Bo Li, Zhongjiang Yan, Mao Yang, and Xiaoya Zuo \\ School of Electronics and Information \\ Northwestern Polytechnical University \\ Xi'an, China \\ Email: yangboo@mail.nwpu.edu.cn, \{libo.npu, zhjyan, yangmao, zuoxy\}@nwpu.edu.cn
}

\begin{abstract}
The multi-channel MAC protocols have been proposed recently to improve the network capacity by accommodating more concurrent transmissions. In this paper, we propose a distributed multi-channel MAC protocol using reliable multiple channel reservation with only a single transceiver. Specifically, the control handshake information is reserved to be re-broadcasted over the control channel to address the multi-channel hidden terminal problem. Besides, by reserving multiple data transmission opportunities on the selected data channel, the control channel congestion is further relieved. Then we prove that the multi-channel hidden terminal problem can be addressed using reservation on the control channel, and analyze the effectiveness of the proposed protocol in the aspect of the average number of data channels simultaneously utilized. Extensive simulation results show that the proposed protocol is able to achieve nearly 2.5 times the saturation throughput of DCA protocol [15] when five-step channel reservation is adopted.
\end{abstract}

Index Terms-Media access control, Channel reservation, Multi-channel.

\section{INTRODUCTION}

With the ever-increasing bandwidth demands of services and applications, wireless media becomes more and more congested. In view of the facts that multiple orthogonal bands are already available (e.g., three orthogonal channels in 2.4 $\mathrm{GHz}$ and 12 channels in $5 \mathrm{GHz}$ band) [1], high throughput can be achieved by establishing parallel transmissions over multiple frequency channels [2], [3]. Since several wireless standards such as IEEE 802.11n [4] and 802.11ac [5] have been proposed to enhance the network performance under the multi-channel condition. However, due to the inflexibility of channel utilization, and the requirement of simultaneously sensing on all-channels per node, performance of the multichannel system is thus restricted. In order to maximize the utilization of multiple available spectrum resources, the design of multi-channel medium access control (McMAC) protocol to coordinate multiple channel access becomes a widely studied research topic [6], particularly in the next generation wireless networks [7], where more devices are deployed densely under the limited spectrum resources condition.
In the current researches of McMAC protocols, the most urgent and critical issue is the multi-channel hidden terminal problem [8], [9], which is caused by selecting an unavailable data channel for transmission, and then results in data collisions. In particular, due to the loss of neighbor nodes control handshake messages, collisions of data transmission between one pair of nodes and the other pair of nodes can not be avoided. The open literatures to address this problem roughly involve time synchronization based protocols [8]- [14] and dedicated control channel (DCC) based protocols [15]- [20].

In time synchronization based McMAC protocols, each node is equipped with one transceiver, and clock synchronization is required. An example is the Multi-channel MAC (MMAC) protocol [9], where time is divided into beacon intervals. All nodes in the network negotiate the data channels over a common channel during the Ad-Hoc Traffic Indication Messages (ATIM) window, and then transmit the following data packets over the selected data channels. However, the multi-channel hidden terminal problem is resolved based on time synchronization, which is a difficult task involving considerable overhead and complexity.

In DCC based McMAC protocols, each node is mainly equipped with two separate half-duplex transceivers, the one transceiver is fixed on the dedicated control channel permanently to contend to access data channels, and the other one switches to the selected data channel to transmit data packets. One representative of this approach is Dynamical Channel Assignment (DCA) protocol [15], which avoids the multi-channel hidden terminal problem by incurring significant hardware cost with the availability of multiple transceivers per device. Moreover, when a relative large number of data channels and nodes are present, this dedicated control channel will be highly congested due to the limited bandwidth, especially when the packets are fairly short [11]. Fortunately, the serious congestion on the dedicated control channel is alleviated effectively in [17], by using multi-step channel reservation scheme with multiple transceivers.

In this paper, a distributed multi-channel MAC protocol by using only a single transceiver is proposed, which is named as $m$-RCR, where $m$ represents the number of steps 
we employ in the channel reservation. In the proposed $m$ RCR protocol, the control handshake information is reserved to be re-broadcasted over the control channel at the appropriate moment, thus the neighbor nodes are able to gather the channel usage information so as to resolve the multi-channel hidden terminal problem. Besides, by reserving multiple data transmission opportunities on the selected data channel with only one successful control handshake, the congestion on the control channel is further relieved. The main contributions of this paper can be summarized as follows:

- We present a novel multi-channel MAC protocol to coordinate multiple channel access using a single transceiver without the requirements for time synchronization.

- The multi-channel hidden terminal problem is addressed by using the reservation on the control channel, which is theoretically proved to be feasible.

- The control channel congestion is relieved by using multi-step reservations on the data channels, and the average number of data channels simultaneously utilized is theoretically analyzed.

- Extensive simulation results demonstrate that the saturation throughput of the proposed protocol is nearly 2.5 times compared with that of the traditional DCA when five-step channel reservation is adopted.

The remainder of the paper is organized as follows. First, the system model is described in Section II. Then in Section III, the operational details of the proposed $m$-RCR protocol is presented, which is followed by the theoretical analysis given in Section IV. In Section V, performance evaluation is presented to demonstrate the effectiveness of our proposed protocol. Finally, some conclusions are drawn in Section VI.

\section{SySTEM MODEL}

We consider a distributed network, which consists of $N$ contending nodes. Each node is equipped with a single halfduplex transceiver, each transceiver is able to switch among the control channel and all the data channels. We assume that there are $K+1$ orthogonal frequency channels with equal bandwidth. One of the channels is configured as the control channel $(\mathrm{CH})$, and the other $K$ channels are configured as data channels, denoted as $D H_{i}, i=1, \ldots, K$. Moreover, it is assumed that the size of all the data packets in the networks is identical, and all the channel conditions are ideal. Therefore, no channel transmission errors exists.

For the sake of our protocol design, we modify the control packets by adding some new fields based on the traditional IEEE 802.11 Distributed Coordination Function (DCF).

The request-to-send (RTS) packet has four new fields: a) the available channel list $(A C L)$, which contains the current available data channels of its own; b) the number of reservation steps $(\mathrm{m})$; c) the time cycle of the reservation on the control channel $\left(T_{C}\right)$; and d) the time cycle of the reservation over data channels $\left(T_{D}\right)$. The RTS packet is represented as $R T S\left(A C L, m, T_{C}, T_{D}\right)$.

The clear-to-send (CTS) packet also has four new fields: a) the number of reservation steps $(m)$; b) the time cycle of the reservation on the control channel $\left.\left(T_{C}\right) ; \mathrm{c}\right)$ the time cycle of the reservation over data channels $\left(T_{D}\right)$; and d) the index of selected data channel $\left(D H_{S}\right)$. The CTS packet is represented as $C T S\left(D H_{S}, m, T_{C}, T_{D}\right)$, which is transmitted over the control channel to inhibit its neighborhood from using the same data channel.

The reservation (RES) packet is constructed and transmitted over the control channel to confirm the channel reservation, which is also used to inhibit its neighborhood from using the same data channel. The structure of RES packet is similar to the CTS, it can be represented as $\operatorname{RES}\left(D H_{S}, m, T_{C}, T_{D}\right)$. Furthermore, compared with DCA protocol, there are three new additional items in our proposed $m$-RCR protocol, including $m, T_{C}$ and $T_{D}$.

Without loss of generality, suppose that each control channel handshake is preceded by a random back-off procedure, which is expressed as $t_{B K} \cdot t_{C} \triangleq t_{R T S}+t_{C T S}+t_{R E S}+2 t_{S I F S}$ represents the duration of a successful handshake on the control channel, and $t_{D} \triangleq t_{D a t a}+t_{S I F S}+t_{A C K}$ is the duration of a data transmission. Moreover, each node pair turns back to the control channel to carry on virtual carrier sensing after finishing each reserved data transmission. The duration of carrier sensing on the control channel is represented as $\mathbb{R}$.

\section{The Proposed $m$-RCR PROTOCOL}

The basic idea of our proposed $m$-RCR protocol consists of two aspects. First, the RES packet is reserved to be rebroadcasted over the control channel at the appropriate time, to address the multi-channel hidden terminal problem. Next, multiple data transmission opportunities are reserved in advance on the selected data channel with only one success control handshake, the control channel congestion is then relieved.

The proposed protocol contains four stages, which are illustrated in Fig. 1.

- Control handshake. The source node and destination node exchange the control packets (including the RTS/CTS/RES packets) over the dedicated control channel, to negotiate the frequency channel for the following data transmissions.

- Reservation on the control channel. The RES packet is reserved to be re-broadcasted by both the source node and the destination node over the control channel, respectively. Therefore, the control handshake information is guaranteed to be received by the neighbor nodes, then the multi-channel hidden terminal problem is addressed.

- Multi-step reservations on the data channels. Multiple data transmission opportunities are reserved in advance with only one success control handshake on the selected data channel, thus the control channel congestion is effectively relieved.

- Collision avoidance. A new handshake process over the control channel will not start until waiting a period of time, to avoid the collisions with the neighbor nodes.

As a reference, the pseudo codes of the source node and destination node are given in Algo.1 and Algo.2, respectively. 
Specifically, for the source node in Algo.1, the control handshake mainly consists of line $2 \sim$ line 6 , reservation on the control channel mainly consists of line $7 \sim$ line 11, multi-step reservations on the data channels mainly consist of line $12 \sim$ line 33, and finally the collision avoidance mainly consists of line $34 \sim$ line 40 . Moreover, for the destination node in Algo.2, the control handshake mainly consists of line $1 \sim$ line 5 , reservation on the control channel mainly consists of line $6 \sim$ line 8 , and finally multi-step reservations on the data channels mainly consist of line $9 \sim$ line 24 .

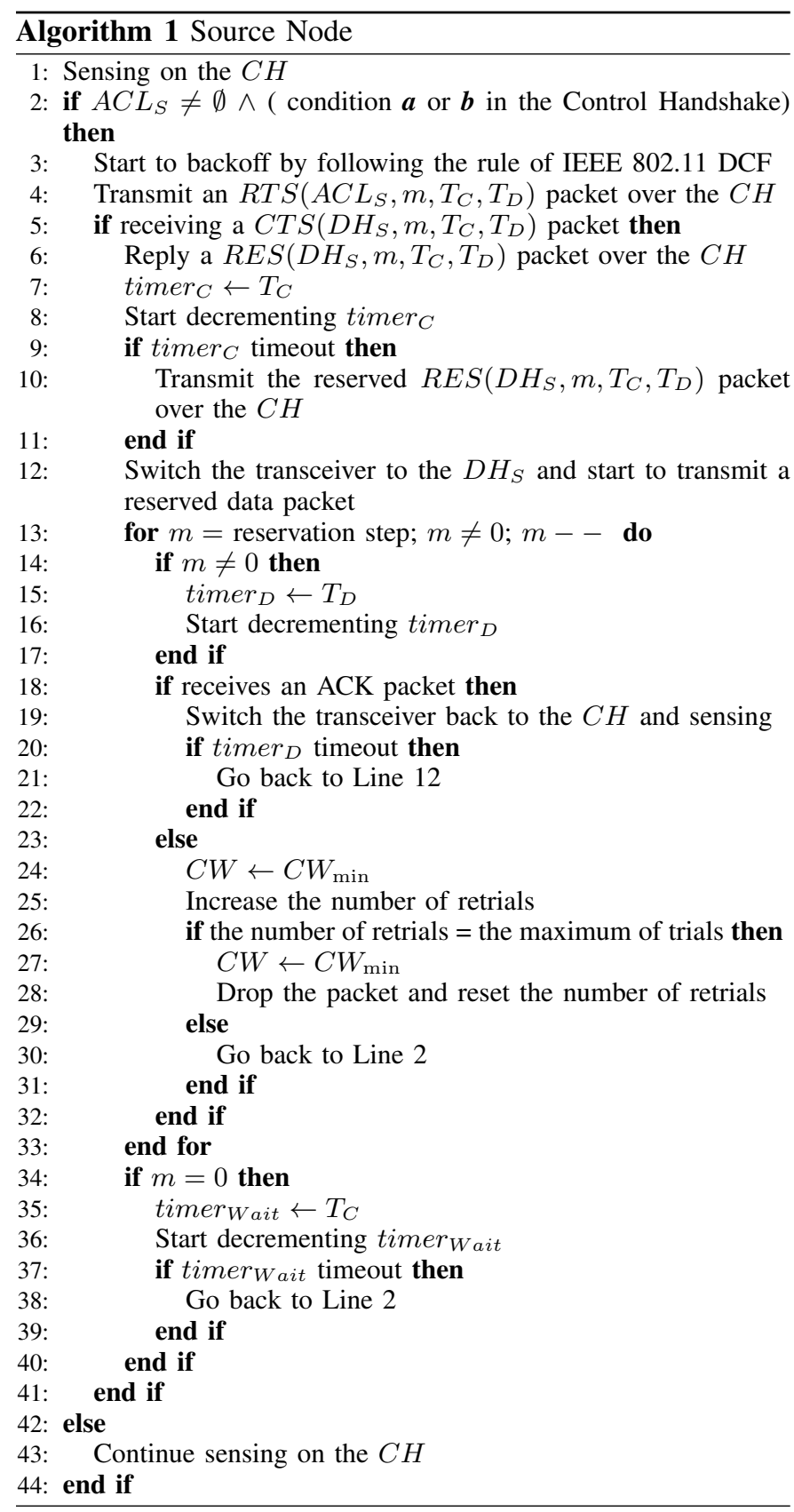

\section{A. Control handshake}

Suppose that a source node ' $\mathrm{S}$ ' wants to transmit data packets to its destination node ' $\mathrm{D}$ '. At least one of the fol-

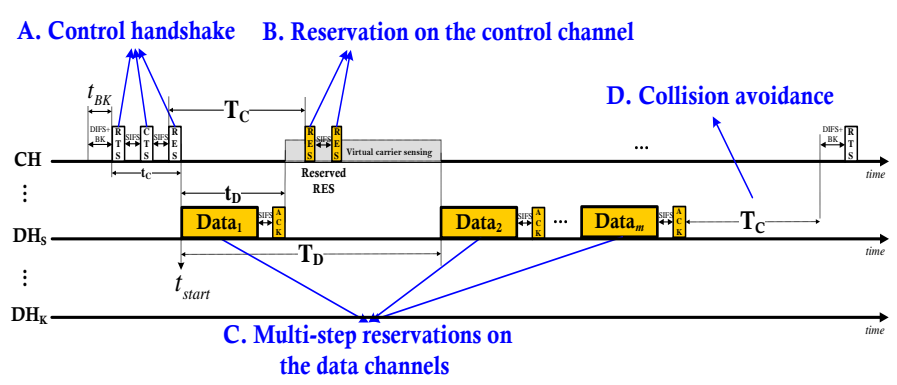

Fig. 1. The operation of $m$-RCR protocol. The process contains four stages: Control handshake (including RTS/CTS/RES packets exchange), Reservation on the control channel (the RES packet is reserved to re-broadcast on the control channel by both of the source node and destination node, respectively), Multi-step reservations on the data channels (multiple data transmissions are reserved with only one successful control handshake), and Collision avoidance (a new control channel handshake will not be initiated until after a period of time).

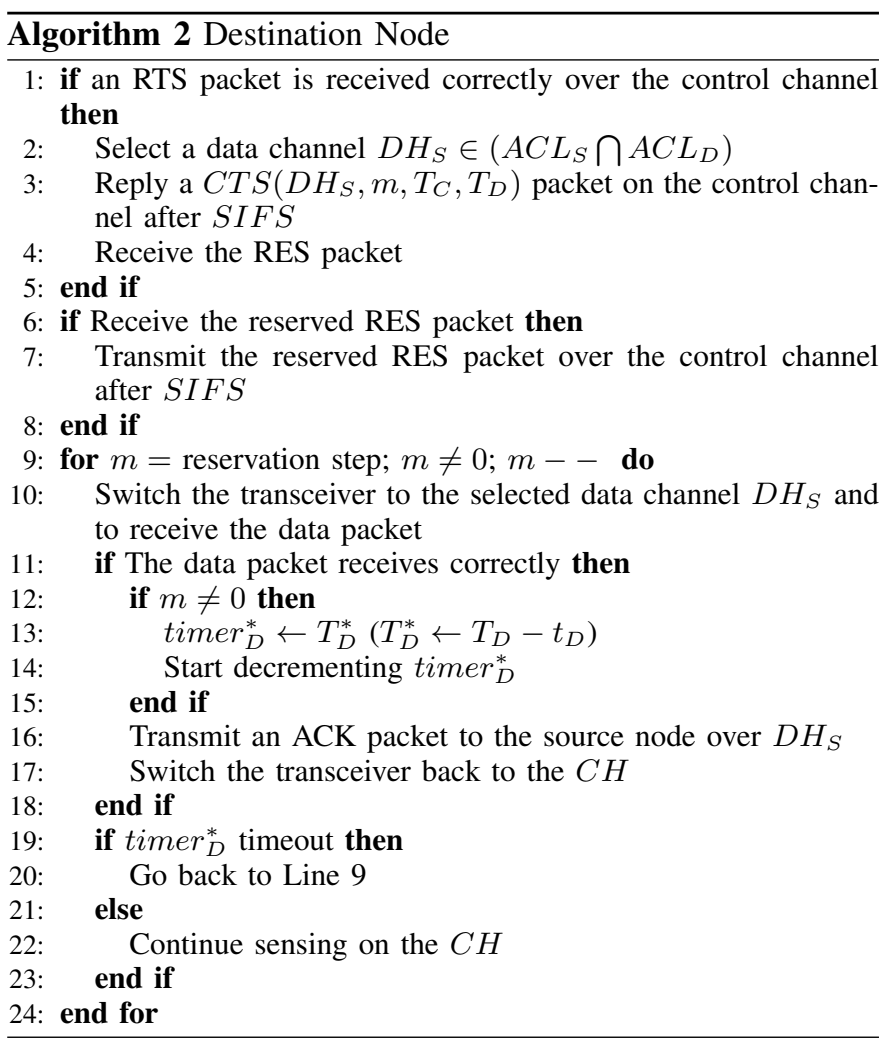

lowing two conditions should first be satisfied: $\boldsymbol{a}$ ) the number of yet-to-be-sent data packets is no less than a threshold, which means that there are enough data packets waiting in the queue, $\boldsymbol{b}$ ) the maximum queuing delay of data packets is larger than a tolerable value, which indicates that the packets waiting in the queue should be transmitted as soon as possible. If one of the two conditions is satisfied, node ' $S$ ' searches for all the candidate data channels and records in its available channel list $A C L_{S}$, and then transmits an $R T S\left(A C L_{S}, m, T_{C}, T_{D}\right)$ packet to node 'D' after a successful competition over the control channel if $A C L_{S} \neq \emptyset$. 


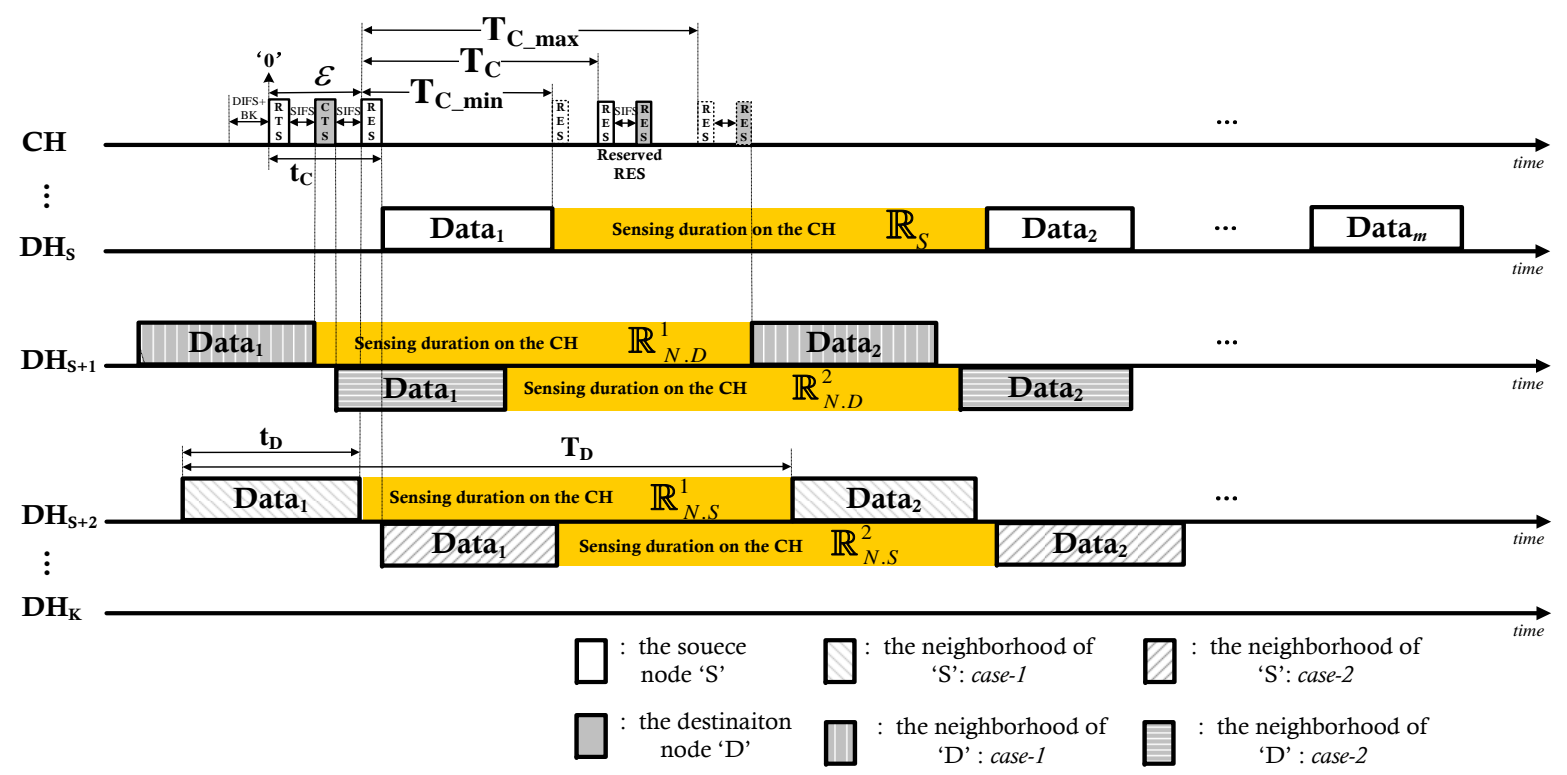

Fig. 2. The analysis of $T_{C}$. Suppose the neighborhood of the destination node and the neighborhood of the source node transmit data packets over the data channel $\mathrm{DH}_{S+1}$ and $\mathrm{DH}_{S+2}$, respectively. The missed control handshake information can be received by all of the neighbor nodes only if the transmission time of the two reserved RES packets within the intersection of $\mathbb{R}_{N . S}, \mathbb{R}_{N . D}$ and $\mathbb{R}_{S}$. The detailed proof is presented in Section IV.

On receiving the RTS packet, node ' $D$ ' selects one of the common available data channels $D H_{S} \in\left(A C L_{S} \bigcap A C L_{D}\right)$, and then replies with a $C T S\left(D H_{S}, m, T_{C}, T_{D}\right)$ packet. After receiving the CTS packet successfully, node ' $\mathrm{S}$ ' transmits a $R E S\left(D H_{S}, m, T_{C}, T_{D}\right)$ packet to confirm the channel reservation.

\section{B. Reservation on the control channel}

After finishing the control handshake successfully, the RES packet is reserved to be re-broadcasted by node ' $\mathrm{S}$ ' over $\mathrm{CH}$ according to the time period of $T_{C}$, where $T_{C} \in\left[t_{R E S}+\right.$ $\left.t_{D}, \quad T_{D}-t_{D}-t_{C T S}-2 t_{R E S}-2 t_{S I F S}\right]$ and $T_{D} \geq 2 t_{D}+$ $3 t_{R E S}+2 t_{S I F S}+t_{C T S}$. The theoretical proof is given in section IV. Receiving the RES, node ' $D$ ' re-broadcasts the RES packet over the control channel after SIFS, to inhibit its neighborhood from using the same data channel.

\section{Multi-step reservations on the data channels}

Meanwhile, $m$ data transmission opportunities are reserved in advance with only one successful control handshake, i.e., the reserved time instants are $t_{\text {start }}+i \times T_{D}, i=0,1, \ldots, m-$ 1 , as shown in Fig. $1 . m$ is the reservation steps, and $t_{\text {start }}$ represents the time instant to transmit the first reserved data packet.

\section{Collision avoidance}

Finally, when the last reserved data packet is transmitted, node ' $S$ ' will not start to initiate a new handshake process over the control channel until its waiting a time period of $T_{C}$, so as to prevent from colliding with the reserved RES packet of its neighbors.

\section{THEORETICAL ANALYSIS}

In this section, we prove that the multi-channel hidden terminal problem can be addressed by using reservation on the control channel in Theorem 1, where the range of $T_{C}$ is derived. Then the average number of data channels simultaneously utilized is analysed in Theorem 2, which reflects the "capacity" for the multi-channel system from the theoretical point of view.

Theorem 1. Suppose that all the channel conditions are ideal, thus no channel transmission errors exists. If $T_{C} \in\left[t_{R E S}+\right.$ $\left.t_{D}, T_{D}-t_{D}-t_{C T S}-2 t_{R E S}-2 t_{S I F S}\right]$, where $T_{D} \geq 2 t_{D}+$ $3 t_{R E S}+2 t_{S I F S}+t_{C T S}$, then the control channel handshake information can be correctly received by all of the neighbors.

Proof: All of the neighbor nodes can be classified into two categories: the neighborhood of the destination node (e.g., node ' $\mathrm{D}$ '), and the neighborhood of the source node (e.g., node ' $S$ '). The missing of control handshake information is caused by the simultaneous data transmission over the other channels. Suppose the neighborhood of the destination node and the neighborhood of the source node are simultaneously transmitting data packets over the data channel $D H_{S+1}$ and $D H_{S+2}$, respectively. To guarantee the control channel handshake information be received by all of the neighbors, we should make sure that the neighborhood of node ' $\mathrm{D}$ ' and the neighborhood of node ' $S$ ' receive the reserved information over the control channel independently. Therefore, we analyze two extreme cases, i.e., the data transmission of the neighborhood just finishes at the beginning of the handshake (e.g., the CTS/RES packets), or the data transmission just starts at the end of the handshake, as illustrated in Fig. 2. 
Denote $\mathbb{R}_{N . S}^{1}, \mathbb{R}_{N . S}^{2}, \mathbb{R}_{N . D}^{1}$ and $\mathbb{R}_{N . D}^{2}$ as the sensing durations of the neighborhood of the source node and the neighborhood of the destination node in two extreme cases, respectively. Consider the moment of transmitting RTS packet as the starting time, then we have

$$
\begin{aligned}
& \mathbb{R}_{N . D}^{1}=\left[t_{R T S}+t_{S I F S}, t_{R T S}+t_{S I F S}+\Delta\right] \\
& \mathbb{R}_{N . D}^{2}=\left[t_{R T S}+t_{S I F S}+t_{C T S}+t_{D}, t_{R T S}+t_{S I F S}+\right. \\
& \left.t_{C T S}+t_{D}+\Delta\right] \\
& \mathbb{R}_{N . S}^{1}=\left[t_{R T S}+2 t_{S I F S}+t_{C T S}, t_{R T S}+2 t_{S I F S}+t_{C T S}\right. \\
& +\Delta] \\
& \mathbb{R}_{N . S}^{2}=\left[t_{C}+t_{D}, t_{C}+t_{D}+\Delta\right],
\end{aligned}
$$

where $\Delta=T_{D}-t_{D}$. Then the overlapped sensing durations can be obtained as

$$
\begin{aligned}
& \mathbb{R}_{N . D}=\mathbb{R}_{N . D}^{1} \cap \mathbb{R}_{N . D}^{2} \\
& =\left[t_{R T S}+t_{S I F S}+t_{C T S}+t_{D}, t_{R T S}+t_{S I F S}+\Delta\right] \\
& \mathbb{R}_{N . S}=\mathbb{R}_{N . S}^{1} \cap \mathbb{R}_{N . S}^{2} \\
& =\left[t_{C}+t_{D}, t_{R T S}+2 t_{S I F S}+t_{C T S}+\Delta\right] .
\end{aligned}
$$

Moreover, denote $\mathbb{R}_{S}$ as the sensing duration over the control channel of the source node, where $\mathbb{R}_{S}=\left[t_{C}+t_{D}, t_{C}+\right.$ $\left.t_{D}+\Delta\right]$. Then the missed control handshake information can be received by all of the neighbor nodes only if the transmission time of the two reserved RES packets is within the intersection of $\mathbb{R}_{N . S}, \mathbb{R}_{N . D}$ and $\mathbb{R}_{S}$. According to Fig. 2, we have

$$
\mathbb{R}_{S} \cap \mathbb{R}_{N . S} \cap \mathbb{R}_{N . D}=\left[t_{C}+t_{D}, t_{R T S}+t_{S I F S}+\Delta\right] .
$$

Thus the range of $T_{C}$ is derived as

$$
\begin{aligned}
& {\left[T_{C}^{\min }, T_{C}^{\max }\right]} \\
& =\left[t_{C}+t_{D}-\varepsilon, t_{R T S}+t_{S I F S}+\Delta-\varepsilon-2 t_{R E S}-t_{S I F S}\right] \\
& =\left[t_{R E S}+t_{D}, T_{D}-t_{D}-t_{C T S}-2 t_{R E S}-2 t_{S I F S}\right] .
\end{aligned}
$$

Where $\varepsilon=t_{R T S}+t_{C T S}+2 t_{S I F S}$. Besides, considering the inequality relations of $T_{C}^{\max } \geq T_{C}^{\min }$ should be satisfied, we can further obtain that $T_{D} \geq 2 t_{D}+3 t_{R E S}+2 t_{S I F S}+$ $t_{C T S}$. Then the control channel handshake information can be received by all of the neighbors and the inappropriate data negotiation is accordingly avoided. Therefore, the multichannel hidden terminal problem is finally addressed.

Theorem 2. Let $\mathcal{N}_{D C A}$ and $\mathcal{N}_{R C R}$ be the average number of data channels simultaneously utilized in DCA and our proposed $m$-RCR protocol, respectively, then we have

$$
\mathcal{N}_{R C R} \stackrel{\widetilde{\leq}}{\leq} m \cdot \mathcal{N}_{D C A} .
$$

Where the symbol " $\simeq$ " means that it is an approximated upper bound.

Proof: Denote $\overline{t_{B K}^{D C A}}$ and $\overline{t_{B K}^{R C R}}$ as the average duration of the random back-off procedure in DCA and $m$-RCR protocols. Then if a bottleneck problem happens in DCA, the average number of data channels can be simultaneously utilized [11] can be obtained as

$$
\mathcal{N}_{D C A} \triangleq \frac{t_{D}}{\overline{t_{B K}^{D C A}}+t_{C}^{D C A}},
$$

where $t_{C}^{D C A}$ and $t_{D}$ represent the duration of a successful control handshake in DCA and the duration of a data transmission. In addition, because $m$ data transmission opportunities are reserved in $m$-RCR with only one successful control handshake, then we can obtain

$$
\mathcal{N}_{R C R} \triangleq \frac{m \cdot t_{D}}{\overline{t_{B K}^{R C R}}+t_{C}^{R C R}},
$$

where $t_{C}^{R C R}$ represents the duration of a successful control handshake in $m$-RCR protocol. Due to the little complements of the control packets based on DCA, then we have $t_{C}^{R C R} \approx t_{C}^{D C A}$. By using the reservation on the control channel in $m$-RCR, the RES packet is re-broadcasted over the control channel, then the average duration of random back-off procedure is enlarged, i.e., $\overline{t_{B K}^{R C R}} \geq \overline{t_{B K}^{D C A}}$, where the equality normally holds under the few contentions condition. Therefore, we can finally obtain

$\mathcal{N}_{R C R}=\frac{m \cdot t_{D}}{\overline{t_{B K}^{R C R}}+t_{C}^{R C R}} \stackrel{\widetilde{z}}{\leq} m \cdot \frac{t_{D}}{\overline{t_{B K}^{D C A}}+t_{C}^{D C A}}=m \cdot \mathcal{N}_{D C A}$.

This completes the proof.

It can be seen that compared with DCA protocol, the number of data channels simultaneously utilized in $m$-RCR is improved by close to $m$ times, which effectively relieves the control channel congestion.

\section{Performance Evaluation}

In our simulations, we consider a single-hop wireless network, in which all nodes are within the reach of each other's transmission range. All channels are assumed to be ideal, in the sense that no channel transmission errors exists. There are 50 contending nodes (50 flows) in the network, and each flow is with the constant bit rate (CBR) traffic, where the packet size is 1024 bytes. Some other simulation parameters are given in Table I.

TABLE I. Simulation Parameters.

\begin{tabular}{l|l}
\hline Parameters & Values \\
\hline \hline Transmission rate for data channels & $11 \mathrm{Mbps}$ \\
\hline Transmission rate for the control channel & $2 \mathrm{Mbps}$ \\
\hline$C W_{\min }$ & 15 \\
\hline$C W_{\max }$ & 1023 \\
\hline SIFS & $10 \mu \mathrm{s}$ \\
\hline DIFS & $50 \mu \mathrm{s}$ \\
\hline Slot time & $20 \mu \mathrm{s}$ \\
\hline The MAC queue length & 50 \\
\hline$T_{C}$ & $1 \mathrm{~ms}$ \\
\hline$T_{D}$ & $7 \mathrm{~ms}$ \\
\hline \hline
\end{tabular}

Compared with IEEE 802.11 DCF and DCA protocols, the performance of $m$-RCR is evaluated by extensive simulations using ns-2 simulator [21] (ns-2.33), in terms of the aggregate throughput and the average packet delay.

Fig. 3 shows that the aggregate throughput achieved by IEEE 802.11 DCF, DCA and our proposed $m$-RCR protocols, 


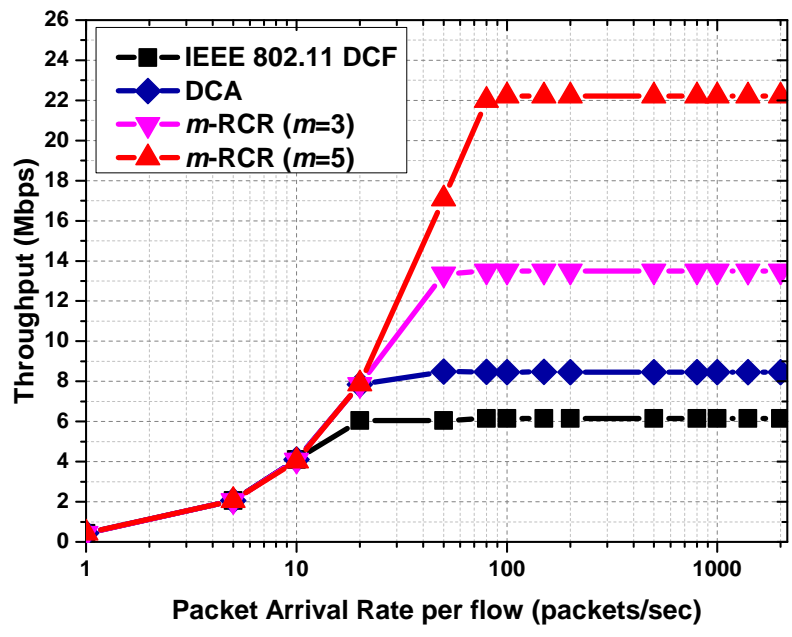

Fig. 3. Aggregate throughput versus packet arrival rate per flow.

respectively. In Fig. 3, we consider that there are one control channel and ten data channels. It can be seen that the system throughput is increased as the traffic rate increases, and $m$ RCR obtains higher throughput than DCA. This is mainly because more data channels can be utilized to bear the everincreasing data traffic, which can be verified by checking Fig. 4. Therefore, the control channel congestion is alleviated, and the system throughput is improved, especially when reservation step is appropriately large. We can also see that the saturation throughput of $m$-RCR is almost 2.5 times of that of DCA when five-step reservations on the data channels is adopted.

Fig. 4 shows the system throughput versus the number of data channels under the saturation traffic condition. It can be seen that due to the capability of utilizing multiple channels, the network throughput of DCA and $m$-RCR are both improved when the number of data channels increases. Furthermore, the control channel has been already saturated in DCA when just two data channels are used, thus the system

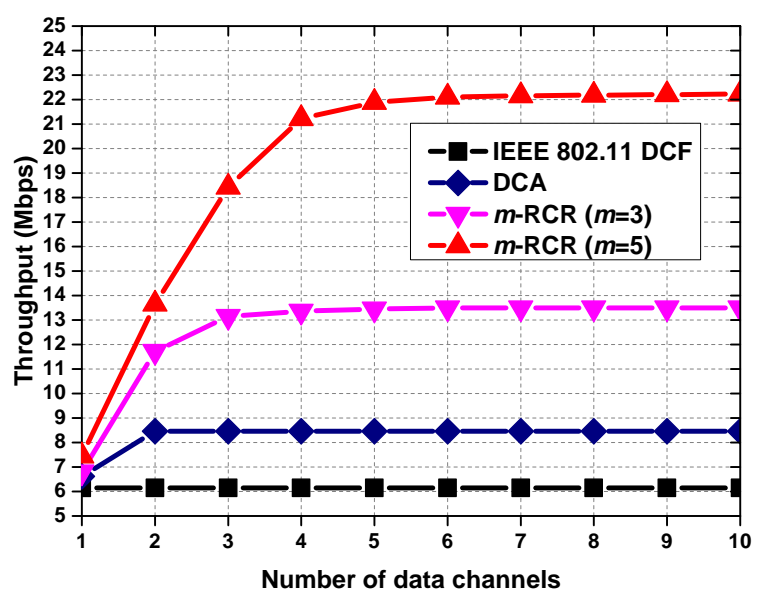

Fig. 4. Throughput versus number of data channels.

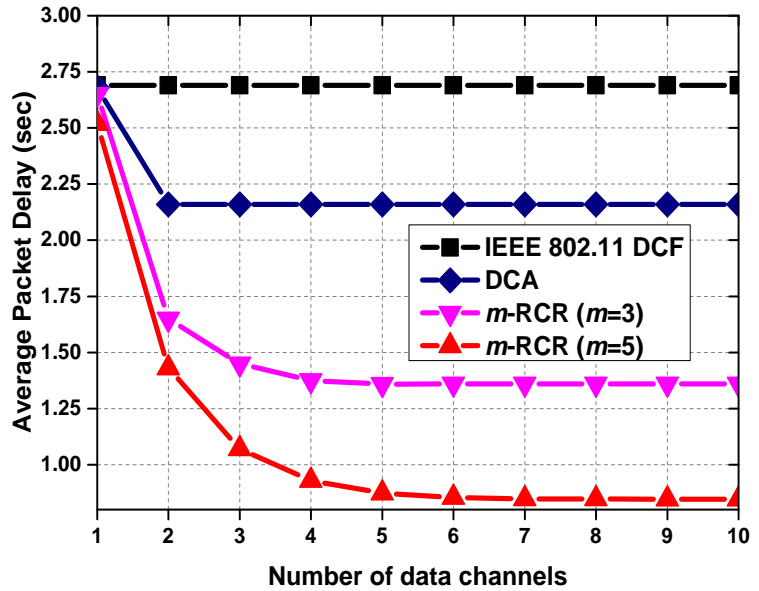

Fig. 5. Average packet delay versus number of data channels.

throughput is restricted. Conversely, almost eight data channels can be utilized in $m$-RCR protocol by using five-step channel reservation, which coincides with the Theorem 2.

Finally, the average packet delay for different number of data channels is illustrated in Fig. 5. When the number of data channels increases, the average packet delay decreases for both DCA and $m$-RCR protocols, and $m$-RCR obtains much lower delay when the reservation step is appropriately large. This is mainly because that multiple data transmission periods are reserved in advance in $m$-RCR, then the following data packets are guaranteed to be transmitted on time without collisions.

\section{CONCLUSION}

In this paper, we propose a distributed multi-channel MAC protocol, i.e., $m$-RCR, which exploits multi-channel reservation to coordinate channel access by using only a single transceiver. The multi-channel hidden terminal problem is addressed based on reservation on the control channel, and the control channel congestion is relieved by reserving multiple data transmission opportunities in advance. Extensive simulations demonstrate that the saturation throughput of $m$-RCR is close to 2.5 times of that of DCA when five-step channel reservation is adopted.

\section{ACKNOWLEDGMENT}

This work is supported in part by the National Natural Science Foundations of CHINA (Grant No. 61271279, and 61201157), the National 863 plans project (Grant No. 2014AA01A707, and 2015AA011307), the National Science and Technology Major Project (Grant No. 2015ZX03002006), and the Fundamental Research Funds for the Central Universities (Grant No. 3102015ZY038, 3102015ZY039).

\section{REFERENCES}

[1] Wireless LAN medium access control (MAC) and physical layer (PHY) specifications, IEEE standard 802.11, Aug. 1999.

[2] Cetinkaya, C., "Multi-Channel Cooperative MAC Protocol for Wireless LANs," Ad Hoc Networks, pp. 1-21, 2015. 
[3] Almotairi, K.H. and Shen, X.S., "A Distributed Multi-Channel MAC Protocol for Ad Hoc Wireless Networks," IEEE Trans. Mobile Comput., vol. 14, no. 1, pp. 1-13, Jan. 2015.

[4] IEEE 802.11n-2009, IEEE Standard for Local and Metropolitan Area Networks 1 Telecommunications and Information Exchange between Systems $\nmid$ Local and Metropolitan Area Networks $¥$ Specific Requirements 1 Part 11: Wireless LAN Medium Access Control (MAC) and Physical Layer (PHY) Specifications Amendment 5: Enhancements for Higher Throughput, 2009.

[5] IEEE 802.11ac/D3.0, Draft Standard for Information Technology 1 Telecommunications and Information Exchange Between Systems C Local and Metropolitan Area Networks 1 Specific Requirements 1 Part 11: Wireless LAN Medium Access Control (MAC) and Physical Layer (PHY) Specifications Amendment 4: Enhancements for Very High Throughput for Operation in Bands Below $6 \mathrm{GHz}$.

[6] J. Mo, H.-S. W. So, and J. Walrand, "Comparison of multichannel MAC protocols," IEEE Trans. Mobile Comput., vol. 7, no. 1, pp. 50-65, Jan. 2008.

[7] Deng, Der-Jiunn, Kwang-Cheng Chen, and Rung-Shiang Cheng, "IEEE 802.11 ax: Next generation wireless local area networks," Heterogeneous Networking for Quality, Reliability, Security and Robustness (QShine), 2014 10th International Conference on. IEEE, pp. 77-82, 2014.

[8] Yan Zhang, Loukas Lazos, Kai Chen, Bocan Hu, and Swetha Shivaramaiah, "FD-MMAC: Combating Multi-Channel Hidden and Exposed Terminals Using a Single Transceiver," in Proc. of IEEE INFOCOM, pp. 2742-2750, 2014.

[9] J. So, N. Vaidya, "Multi-channel MAC for ad hoc networks: handling multi-channel hidden terminals using a single transceiver," in Proc. of MobiHoc, pp. 222-233, 2004.

[10] P. Bahl, R. Chandra, and J. Dunagan, "SSCH: Slotted seeded channel hopping for capacity improvement in IEEE 802.11 ad-hoc wireless networks," in Proc. of ACM MobiCom, pp. 216-230, 2004.
[11] Tie Luo, Motani, M., "Cooperative Asynchronous Multichannel MAC: Design, Analysis, and Implementation," IEEE Trans. Mobile Comput., vol. 8, pp. 338-352, Jan. 2009.

[12] Dang, Duc Ngoc Minh, Choong Seon Hong, and Sungwon Lee, "A hybrid multi-channel MAC protocol for wireless ad hoc networks." Wireless Networks, pp. 1-18, 2014.

[13] J. Zhang, G. Zhou, C. Huang, S.H. Son, and J.A. Stankovic, "TMMAC: An energy efficient multi-channel mac protocol for ad hoc networks," in Proc. of ICC, pp. 3554-3561, 2007.

[14] S. L. Wu and J. Y. Yang, "A novel channel assignment scheme for improving channel reuse efficiency in multi-channel ad hoc wireless networks," Computer Communications, pp. 3416-3424, 2007.

[15] S. L. Wu, C. Y. Lin, Y. C. Tseng and J. P. Sheu, "A New Multi-Channel MAC Protocol with On-Demand Channel Assignment for Multi-Hop Mobile Ad Hoc Networks," in Proc. of I-SPAN, pp. 232-237, 2000.

[16] S.-L. Wu, Y.-C. Tseng, C.-Y. Lin, and J.-P. Sheu, "A multi-channel MAC protocol with power control for multi-hop mobile ad hoc networks," Comput. J., vol. 45, no. 1, pp. 101-110, 2002.

[17] B. Yang, B. Li, Q. Qu, Z. Yan, "A New multi-channel MAC protocol based on multi-step channel reservatio," in Proc. of IEEE ICSPCC, pp. 603-607, 2014.

[18] N. Jain, S.R. Das, and A. Nasipuri, "A multichannel CSMA MAC protocol with receiver-based channel selection for multihop wireless networks," In Proc. of the ICCCN Conference, pp. 432-439, 2001.

[19] Almotairi, K. H., Shen, X., "MMAC-HR: Multi-channel medium access control with hopping reservation for multi-hop wireless," in Proc. of IEEE GLOBECOM, pp. 1-5, 2010.

[20] T. Shu, S. Cui, and M. Krunz, "Medium access control for multichannel parallel transmission in cognitive radio networks," In Proc. of the GLOBECOM, pp. 1-5, 2006.

[21] The Network Simulator ns-2, http://www.isi.edu/nsnam/ns. 